\title{
ANAP

\section{USO DE SISTEMA DE INFORMAÇÃO GEOGRÁFICA PARA MAPEAMENTO DE RECURSOS HÍDRICOS - ESTUDO DE CASO NO PONTAL DO PARANAPANEMA}

\author{
Letícia Aparecida Costa ${ }^{1}$
}

Rebeca Barbosa Boraschi ${ }^{2}$

Alessandra Santos ${ }^{3}$

Ana Paula Marques Ramos ${ }^{4}$

\begin{abstract}
RESUMO
Este trabalho tem como objetivo apresentar o método aplicado para extrair feições geográficas utilizando um Sistema de Informação Geográfica (SIG) e técnicas de Geoprocessamento. A contribuição desse trabalho é disponibilizar dados espaciais de apoio à gestão de recursos hídricos de bacias hidrográficas da Unidade de Gerenciamento de Recursos Hídricos do Pontal do Paranapanema (UGRHI-22). Para tanto foram utilizadas ortografias cedidas pela empresa EMPLASA, na resolução de 1(um) metro e precisão compatível com a escala 1:25.000 e também o software ArcGIS como SIG. Com esses materiais foi possível mapear feições das bacias hidrográficas da UGRHI-22 como cursos d'agua e áreas de nascente, ambas armazenadas em arquivos na extensão shapefile. Para extrair tais feições foi empregada a vetorização manual no ArcGIS, a partir da interpretação visual das ortografias. Dessa forma, foram mapeados aproximadamente $50 \%$ dos cursos d'água da região da UGRHI-22 até o momento. Os resultados desse trabalho proporcionam suporte à delimitação das Áreas de Preservação Permanente e à subsequente manutenção e recuperação de áreas degradadas por ações antrópicas.
\end{abstract}

PALAVRAS-CHAVE: Produção cartográfica. Mapeamento. Recurso hídrico. Planejamento ambiental.

\footnotetext{
1 Engenheira Ambiental, Mestranda em Meio Ambiente e Desenvolvimento Regional pela Universidade do Oeste Paulista (MMADRE/UNOESTE). le costa93@hotmail.com

${ }^{2}$ Graduanda em Engenharia Ambiental, pela Faculdade de Ciências e Tecnologia da Universidade Estadual Paulista (FCT/UNESP). re_boraschi@hotmail.com

3 Graduanda em Engenharia Ambiental e Sanitária pela Universidade do Oeste Paulista. alessandrasantos12@hotmail.com

${ }^{4}$ Engenheira Cartógrafa, Prof ${ }^{\mathrm{a}}$. $\mathrm{Dr}^{\mathrm{a}}$. da Universidade do Oeste Paulista. anaramos@unoeste.br
} 


\title{
USING GEOGRAPHIC INFORMATION SYSTEM FOR MAPPING \\ WATER RESOURCES - CASE STUDY IN THE PONTAL DO \\ PARANAPANEMA
}

\begin{abstract}
This work aims to present the method applied to extract geographic features using a Geographic Information System (GIS) and Geoprocessing techniques. The contribution of this work is providing spatial data to support the management of watersheds water resources of Unidade de Gerenciamento de Recursos Hídricos do Pontal do Paranapanema (UGRHI-22). Therefore it was used orthographies given by the company EMPLASA, with 1(one) meter of resolution and precision compatible with the scale of 1:25,000, also ArcGIS software was used as a SIG. With all these materials it was possible mapping geographic features of watersheds from UGRHI-22 like watercourses and spring areas, both stored on file extension shapefile. To extract features was employed manual vectorization on ArcGIS from the visual interpretation of orthographies. It was mapped about $50 \%$ of the watercourses of the UGRHI-22 region until now. The results of this work may support tasks of delimitation of Permanent Preservation Areas tasks and offer support to the maintenance and recovery of degraded areas by human actions.
\end{abstract}

KEYWORDS: Cartographic process. Mapping. Water resources. Environmental planning.

\section{EL USO DE SISTEMAS DE INFORMACIÓN GEOGRÁFICA PARA EL} MAPEO DE LOS RECURSOS HÍDRICOS - ESTUDIO DE CASO EN EL

\section{PONTAL DE PARANAPANEMA}

\begin{abstract}
RESUMEN
Este trabajo tiene como objetivo exponer el método utilizado para extraer las características geográficas utilizando un Sistema de Información Geográfica (SIG) y técnicas de Geoprocesamiento. La contribución de este trabajo es proporcionar datos espaciales que apoyan la gestión de los recursos hídricos en las cuencas hidrográficas de la Unidad de Gerenciamiento de Recursos Hídricos del Pontal de Paranapanema (UGRHI-22). Por lo tanto, hemos utilizado la ortofotografía proporcionados por la empresa EMLASA, en la resolución de un metro y la precisión compatible con la escala de 1: 25.000 y también el software ArcGIS como SIG. Con estos materiales fue posible mapear características de las cuencas hidrográficas de la UGRHI-22 como los cursos de agua y zonas de nascentes, ambos almacenados en archivos con extensión shapefile. Para extraer tales características fue utilizada la vectorización manual en ArcGIS, a partir de la interpretación visual de la ortofotografía. De este modo, se han mapeado aproximadamente el $50 \%$ de los cursos de agua en la región de UGRHI-22 hasta el momento. Los resultados de este trabajo proporcionan la delimitación de las Áreas de Preservación Permanente y el consecuente mantenimiento posterior y la recuperación de áreas degradadas por la acción humana.
\end{abstract}

PALABRAS CLAVE: Producción cartográfica. Cartografía. Los recursos hídricos. Planificación ambiental.

Revista Científica ANAP Brasi, v. 8, n. 13, 2015, p. 23-37. 


\section{INTRODUÇÃO}

Impactos ambientais são alterações que ocorrem no meio ambiente ou em algum de seus componentes por determinada ação ou atividade humana. Nota-se com isso a necessidade de ferramentas que ofereçam suporte às atividades de conservação, monitoramento $\mathrm{e}(\mathrm{ou})$ de recuperação dos recursos de um ambiente. Um importante instrumento que age nesse sentido é o planejamento ambiental. Segundo Santos (2004), o planejamento ambiental atua, principalmente, como um fator de ordenação do uso e ocupação do meio ambiente, visto que o uso do solo tem relação direta com a degradação do meio.

No contexto de bacia hidrográfica, o planejamento ambiental se constitui em um importante instrumento para auxiliar o gerenciamento dos seus recursos, incluindo-se os recursos hídricos (MOTA, 2003; LEAL, 2012). A concretização do planejamento territorial ambiental necessita ter como alicerce preocupações com a preservação ambiental, conservação de bens naturais e recuperação das áreas degradadas (ROSS, 2009).

O ciclo hidrológico possui sua dinâmica e funcionamento natural, porém, intervenções antrópicas alteram sua naturalidade. Os problemas ocorridos com a água podem ser referentes à quantidade, como escassez, estiagem, ou a qualidade da água, como a contaminação de mananciais que acaba impossibilitando seu uso para abastecimento humano (BRAGA et al., 2005).

Segundo Derisio (2007), os recursos hídricos necessitam ser objeto de um estudo aprofundado, com levantamento e a avaliação de sua qualidade e quantidade desse recurso, uma vez que a água doce utilizável representa menos que 1,1\% da quantidade de água do nosso planeta e essa abundância são espalhadas de forma irregular.

As ações de prevenção ou correção de atividades, naturais ou antrópicas, que ocorrem em um ambiente, em sua maioria, são dependentes do conhecimento 


\section{ANAP

da distribuição espacial das feições desse ambiente, bem como dos fenômenos e de suas relações. Nesse processo, tem papel fundamental, a aplicação dos conceitos de Cartografia e, mais recentemente, de ferramentas computacionais para Geoprocessamento, denominadas de Sistemas de Informação Geográfica (SIG). A ciência cartográfica possibilita a elaboração de produtos referencias que dão suporte ao desenvolvimento de estudos ambientais em quaisquer uma de suas dimensões, tais como inventário, diagnóstico, análise de impacto, políticas de controle da atividade humana, etc. (MEDEIROS; CÂMARA, 2004).

De acordo com Câmara e Davis (2004), o conhecimento sobre a distribuição geográfica dos fenômenos e recursos naturais sempre foi uma parte importante das atividades das sociedades organizadas. Inicialmente, esse conhecimento era obtido apenas em documentos e mapas em papel, o que impedia uma análise espacial a partir da combinação de diversos dados. A partir dos anos 60 , este cenário sofre um processo de transformação, devido ao desenvolvimento da tecnologia de informática, com a qual se torna possível armazenar e representar as informações geográficas em ambiente computacional, dando origem à Cartografia digital e ao Geoprocessamento.

\section{OBJETIVO}

O objetivo desse trabalho é apresentar o método empregado para a extração de feições geográficas em ortofotografias digitais usando um Sistema de Informação Geográfica e técnicas de Geoprocessamento. A principal contribuição desse estudo é disponibilizar dados espaciais de apoio à gestão dos recursos hídricos de bacias hidrográficas da $22^{\text {a }}$ Unidade de Gerenciamento de Recursos Hídricos do Pontal do Paranapanema, a UGRHI-22.

\section{METODODOLOGIA}




\section{ANAP $B_{\text {rasil }}$}

ISSN 1904-3240

v. 8, n. 13

\section{REVISTA CIENTIFICA 2015}

A metodologia deste trabalho está dividida em duas grandes partes: materiais, caracterização da UGRHI-22 e extração das feições geográficas em ortofotografias. Os materiais consistiram em ortofotografias digitais e um Sistema de Informação Geográfica. As ortofotografias foram cedidas pela empresa EMPLASA, a qual é responsável por realizar o projeto de 'Atualização Cartográfica do Estado de São Paulo - Mapeia São Paulo'. Segundo a EMPLASA (2015), tais ortofotos têm resolução espacial de 1 (um) metro $(\mathrm{m})$ e foram produzidas a partir de fotografias aéreas digitais obtidas entre os anos de 2010 e 2011, com resolução espacial de 45 centímetros. Uma média de 40 fotos aéreas foram utilizadas para produzir cada ortofotos. Um total de 1727 ortofotos são necessárias para recobrir todo o Estado de São Paulo. A precisão planimétrica dessas ortofotos digitais é compatível com a escala 1:25.000, e os arquivos são disponibilizados no formato tiff georreferenciado (EMPLASA, 2015). O sistema de informações geográficas utilizado foi o ArcGIS 10.2, o qual é desenvolvido pela empresa americana ESRI.

\section{Caracterização da UGRHI-22}

A $22^{\mathrm{a}}$ Unidade de Gerenciamento de Recursos Hídricos do Pontal do Paranapanema está localizada no extremo oeste do estado de São Paulo, na área limítrofe com os estados do Mato Grosso do Sul e Paraná, entre os rios Paraná e Paranapanema (Figura 1). Além desses, o Santo Anastácio e Pirapozinho são outros dois principais rios dessa unidade de gerenciamento. Um total de 26 municípios estão total ou parcialmente inseridos na UGRHI-22, a qual tem extensão territorial de $11.838 \mathrm{~km}^{2}$ e abrange população superior a 480 mil habitantes (CBH-PP, 2015).

Em razão da diversidade de recursos naturais e múltiplos recursos hídricos presentes na região do Pontal do Paranapanema, vários projetos contemplando a conservação, a recuperação e o monitoramento desses recursos têm sido desenvolvidos com o apoio do governo do Estado de São Paulo. O intuito é o de promover ações nessa unidade hidrográfica, a qual tem a característica de ser formada por várias bacias hidrográficas, como mostra a Figura 2.

Revista Científica ANAP Brasi, v. 8, n. 13, 2015, p. 23-37. 


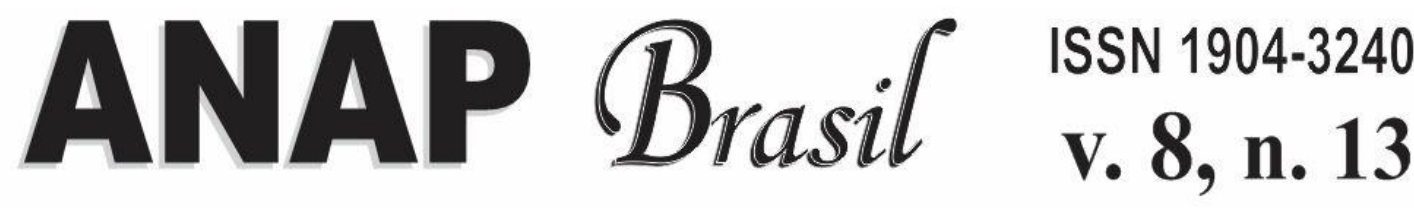

\section{REVISTA CIENTIFICA}

Figura 1: UGRHI-22 e seus municípios.

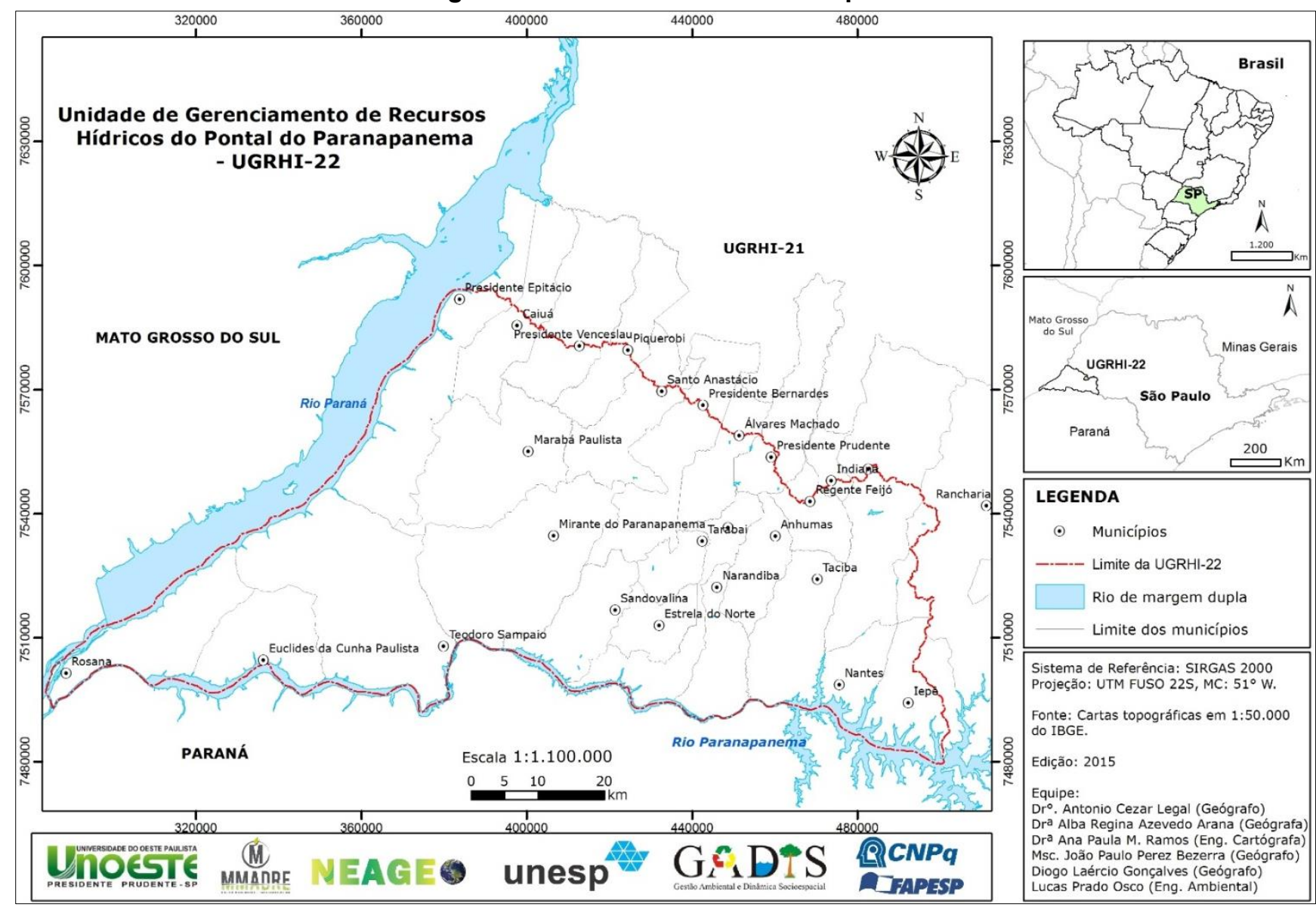

Fonte: Autores

Revista Científica ANAP Brasil, v. 8, n. 13, 2015, p. 23-37. 


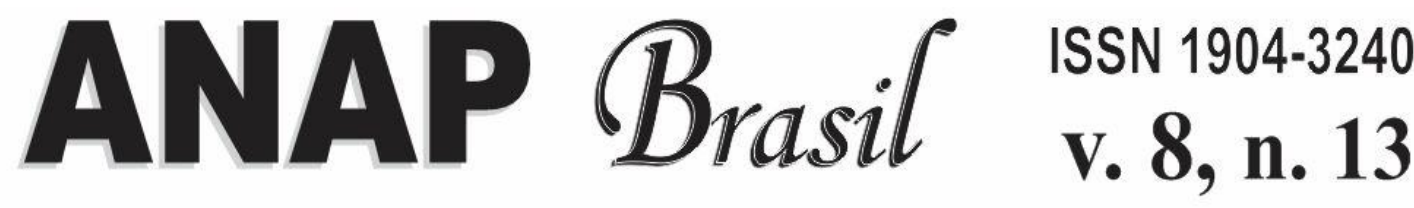

\section{REVISTA CIENTIFICA}

Figura 2: Bacias hidrográficas da UGRHI-22.

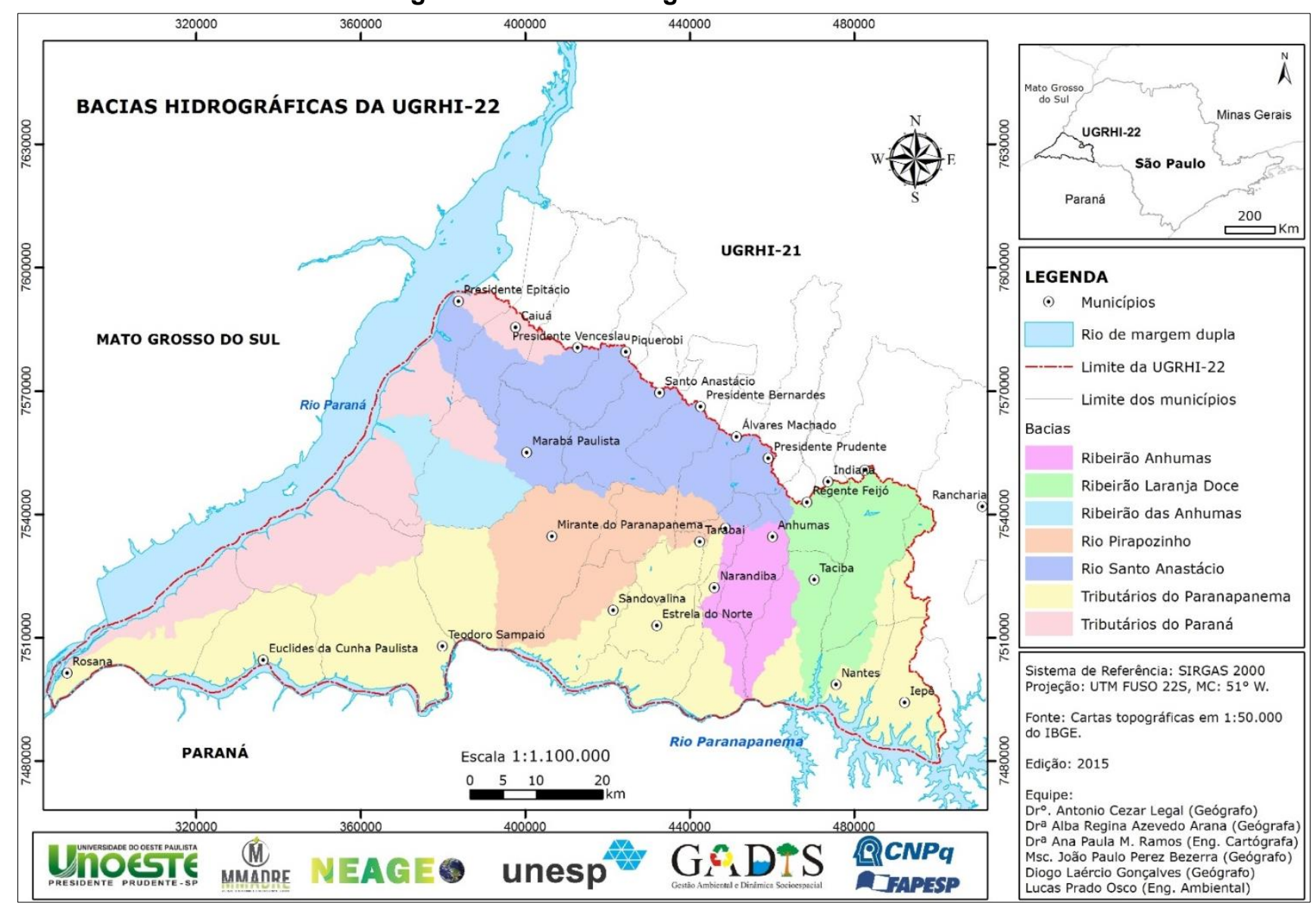

Fonte: Autores

Revista Científica ANAP Brasil, v. 8, n. 13, 2015, p. 23-37. 


\section{ANAP Brasil}

ISSN 1904-3240

V. 8, n. 13

\section{5}

\section{Extração de feições geográficas a partir de ortofotografias digitais}

A atividade de mapeamento das feições da UGRHI-22, teve início no final do segundo semestre de 2014, a partir de uma parceria entre a FCT-UNESP (Faculdade de Ciências e Tecnologia - Universidade Estadual Paulista "Júlio de Mesquita Filho"), por meio do grupo de pesquisa GADIS (Gestão Ambiental e Dinâmica Socioespacial), coordenado pelo Prof. Dr. Antonio Cezar Leal e a Universidade do Oeste Paulista (UNOESTE), por meio do Núcleo de Estudos Ambientais e Geoprocessamento (NEAGEO) e do Programa de Mestrado em Meio Ambiente e Desenvolvendo Regional/MMADRE, dos quais a $\operatorname{Prof}^{\mathrm{a}}$. $\mathrm{Dr}^{\mathrm{a}}$. Ana Paula Marques Ramos é membro. Essa parceria fomenta parte de um Projeto Temático FAPESP (Processo: 2012/23959-9) intitulado "Mapeamento e Análise do Território do Agrohidronegócio Canavieiro no Pontal do Paranapanema-São Paulo-Brasil: Relações de trabalho, conflitos e formas de uso da terra e da água, e a saúde ambiental", coordenado pelo Prof. Dr. Antonio Thomaz Júnior, também, da FCT/UNESP.

As feições que vêm sendo mapeadas nas bacias hidrográficas da UGRHI-22 são cursos d'água e áreas de nascente. Ambas as feições têm sido armazenadas utilizando a primitiva geométrica de polígono, em arquivos na extensão shapefile. $\mathrm{O}$ método empregado para a extração dessas feições consiste na vetorização manual, a partir da interpretação visual das ortofotografias no software ArcGIS. Definiu-se a escala de visualização 1:3.000 para extrair as feições nas ortofotografias, pois estas têm alta resolução espacial $(1 \mathrm{~m})$ e, nesta escala, possibilitam identificar e interpretar mais facilmente as feições geográficas de interesse (Figura 3). 


\section{ANAP

\section{REVISTA CIENTIFICA 2015}

Figura 3: Escala de visualização utilizada para mapear as feições da UGRHI-22.

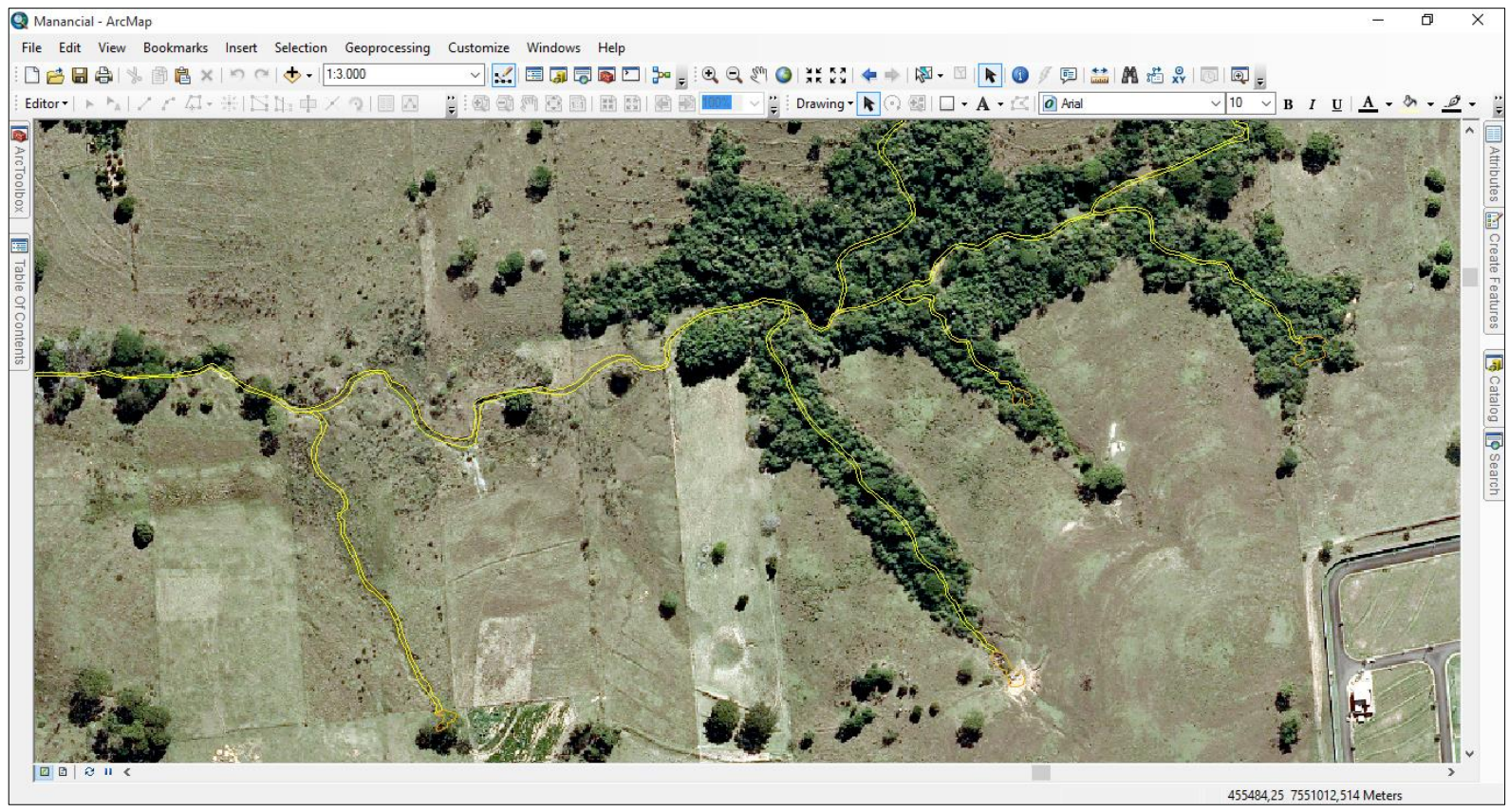

Para atender a diferentes demandas do Projeto Temático FAPESP (Processo: 2012/23959-9), os dados de cursos d'água (em amarelo na Figura 3) e de áreas de nascente (em laranja na Figura 3), inicialmente, obtidos na primitiva geométrica de polígono, estão sendo convertidos para a primitiva geométrica de linha e ponto, respectivamente. Para se obter tais resultados, tem-se recorrido à diferentes ferramentas de Geoprocessamento disponíveis no ArcGIS. No caso das feições de curso d'água, o procedimento aplicado tem consistido em:

1. Aplicação do operador dissolve na primitiva de polígono, para agregar diferentes trechos de cursos d'água em um único;

2. Conversão da primitiva de polígono para a primitiva de linha, por meio da aplicação do operador Polygon to line;

3. Eliminação das extremidades dos polígonos convertidos para linha, por meio pelo operador delete; $\mathrm{e}$

4. Aplicação do operador colapse dual line to centerline na primitiva de linha para se obter o eixo central da camada. 


\section{ANAP $B_{\text {rasil }}$}

ISSN 1904-3240

v. 8, n. 13

No caso das feições de áreas de nascentes, aplicou-se o operador Polygon to line, o qual possibilita obter o centro de um polígono. Com isso, as áreas de nascentes extraídas das ortofotografias na primitiva de polígono puderam ser obtidas como feição pontual.

\section{RESULTADOS}

Para ilustrar a produção cartográfica obtida, até o presente momento no andamento do Projeto Temático FAPESP (Processo: 2012/23959-9), no qual esse trabalho está inserido, a Figura 4 apresenta os cursos d'água já extraídos por fotointerpretação das ortofotografias digitais. Além disso, um estudo de caso é apresentado considerando o Alto Curso do Rio Santo Anastácio, visando ilustrar os resultados parciais obtidos quanto à etapa de conversão dos dados cursos d'água e áreas de nascente para as primitivas geométricas de linha e ponto, respectivamente, como mostra a Figura 5.

Nota-se (Figura 4) que aproximadamente $50 \%$ da região da UGRHI-22 teve os cursos d'água mapeados. Deve-se ressaltar que este é um trabalho oneroso, em termos de tempo, pois requer do operador um treinamento na extração desse tipo de feições por fotointerpretação. Quanto ao método empregado para converter os dados da primitiva de polígono para a primitiva de linha (cursos d'água) e de ponto (nascentes), verifica-se (Figura 6) que o método empregado é eficaz, pois possibilita a obtenção e a manipulação dos dados de drenagem em distintas primitivas. Com isso, esse procedimento deve ser aplicado para todo o restante da UGRHI-22.

Cabe ressaltar que os dados de drenagem podem ser utilizados na delimitação das Áreas de Preservação Permanente, as quais, dentre outras, possibilitam concretizar as ações de manutenção ou de recuperação de áreas degradas por ações antrópicas.

A tomada de decisões para essa área de nosso território é de extrema importância para a manutenção dos recursos hídricos que se encontram 


\section{ANAP

desprotegidos e ameaçados. Com o auxílio do planejamento ambiental é possível efetivar ações que visem a melhoria dessas áreas, visando a proteção dos recursos hídricos. O levantamento de dados sobre os recursos hídricos atua como suporte para o desenvolvimento do planejamento ambiental das áreas em estudo. 


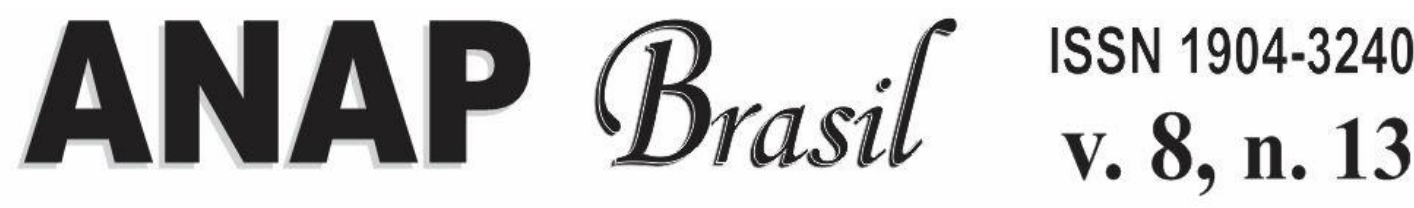

\section{REVISTA CIENTIFICA}

Figura 4: Drenagem extraída das bacias hidrográficas da UGRHI-22.

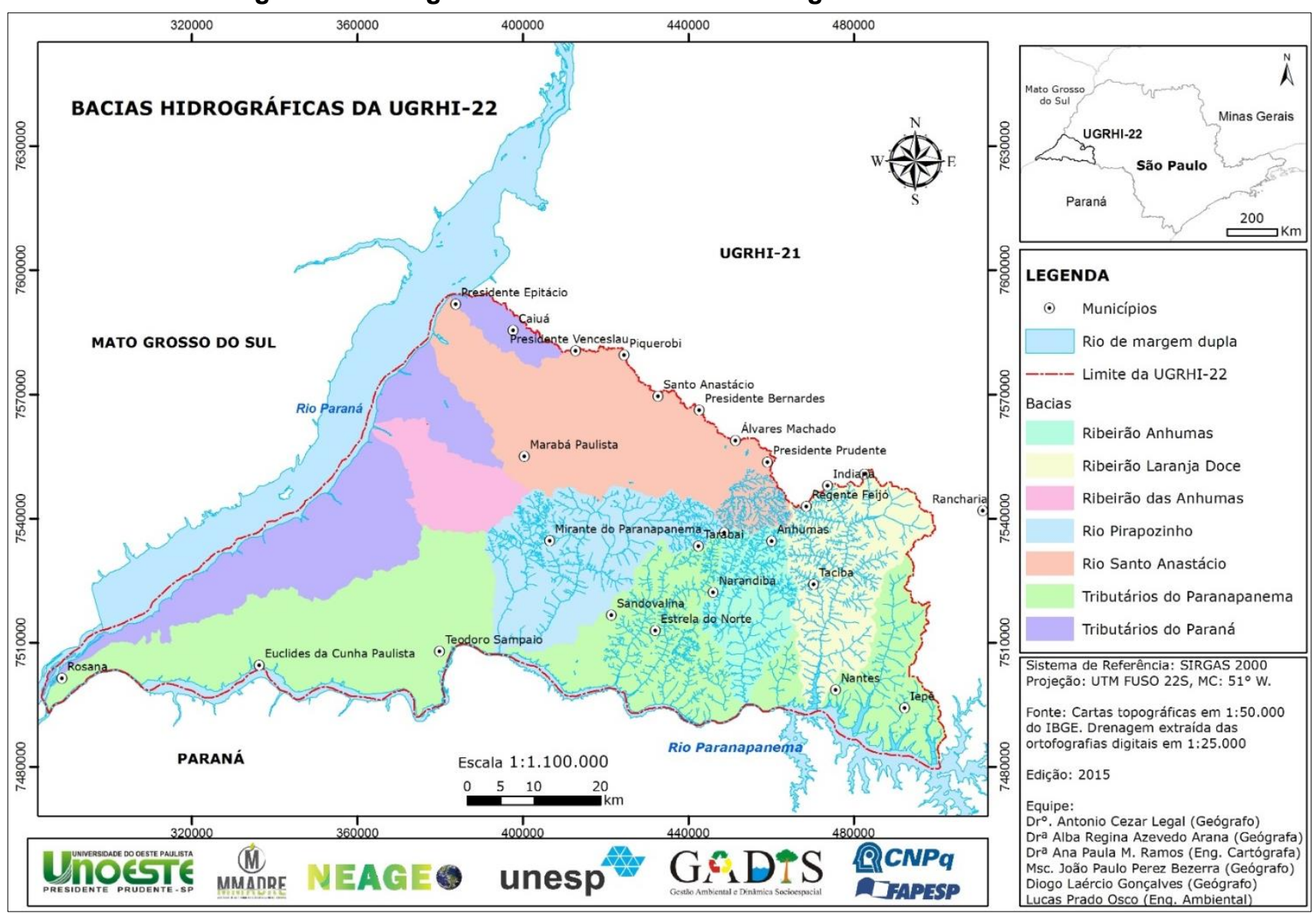

Fonte: Autores.

Revista Científica ANAP Brasd, v. 8, n. 13, 2015, p. 23-37. 


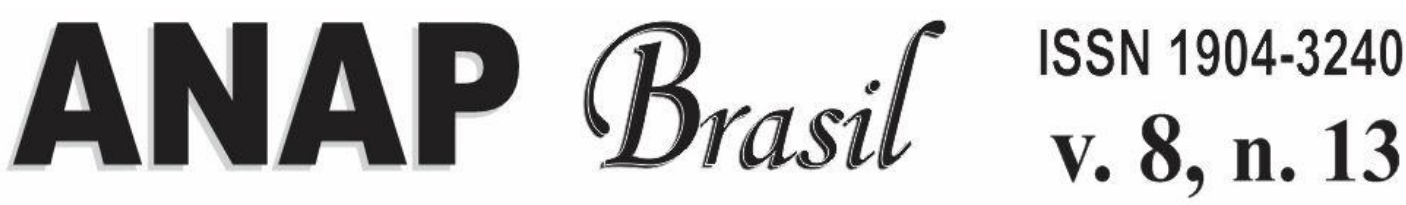

\section{REVISTA CIENTIFICA}

Figura 5: Resultados dos recursos hídricos para o Alto Curso do Rio Santo Anastácio na UGRHI-22.

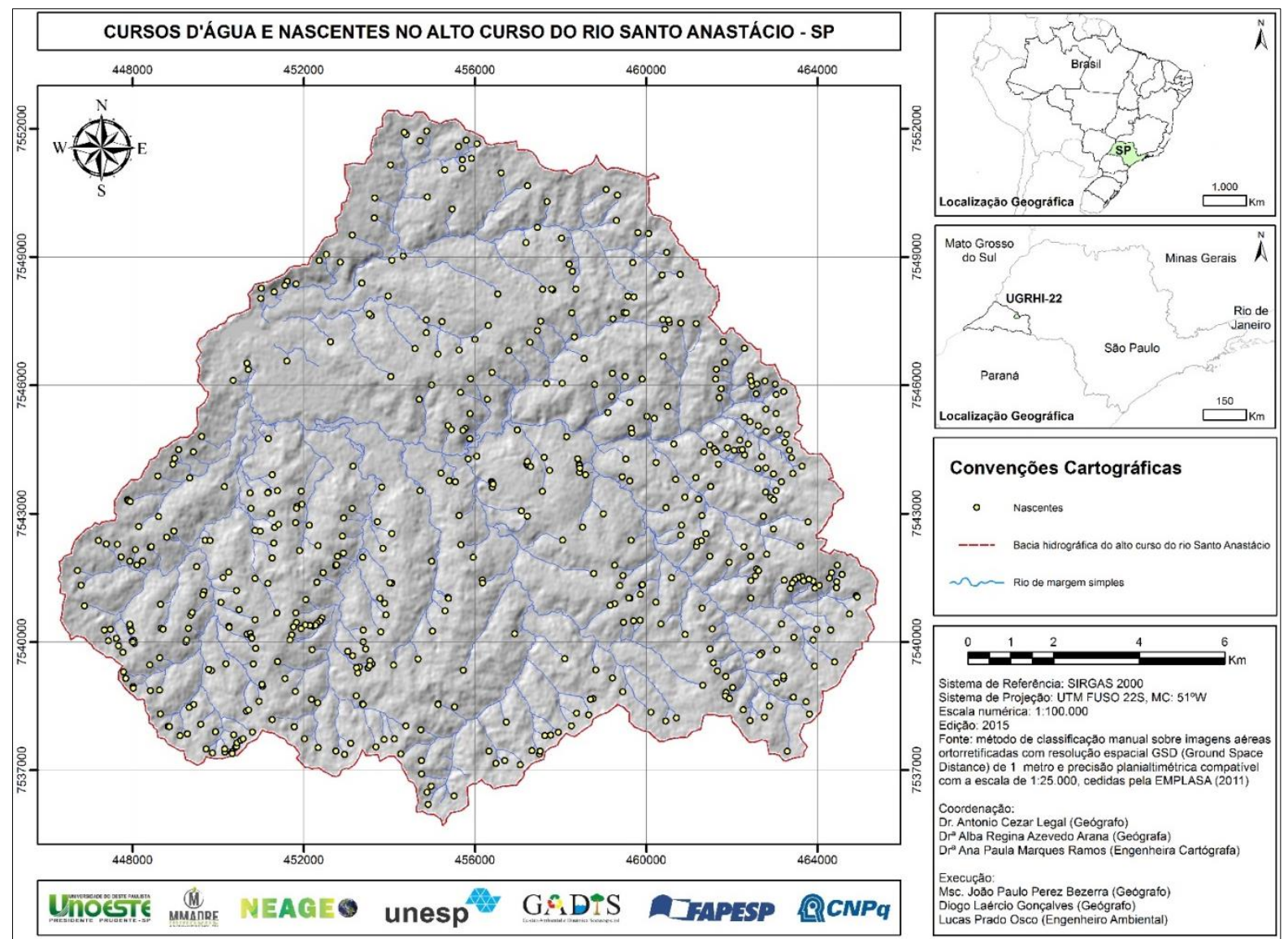

Fonte: Autores

Revista Científica ANAP Brasil, v. 8, n. 13, 2015, p. 23-37. 


\section{ANAP $B_{r a s i l}$ \\ ISSN 1904-3240 \\ v. 8, n. 13}

\section{REVISTA CIENTIFICA}

\section{CONCLUSÃO}

Esse trabalho descreve o método aplicado na extração manual de feições, a partir de ortofotografias digitais, usando um Sistema de Informação Geográfica e técnicas de Geoprocessamento. Conclui-se que os resultados obtidos contribuem para a disponibilização de dados espaciais de apoio à gestão dos recursos das bacias hidrográficas existente na UGRHI-22. Concluise, ainda, que os resultados auxiliam em projetos cuja a temática seja a proteção das águas do Pontal do Paranapanema.

O uso de Sistema de Informação Geográfica e de técnicas de Geoprocessamento se mostraram importantes ferramentas para viabilizar a extração de feições em ortofotografias digitais. Conclui-se que o uso de geotecnologias podem otimizar o desenvolvimento de trabalhos relacionados ao planejamento ambiental de bacias hidrográficas, tais como as presentes na UGRHI-22.

Recomenda-se que os dados resultantes desse trabalho sejam utilizados para promover melhorias na proteção dos recursos hídricos da $22^{\mathrm{a}}$ Unidade de Gerenciamento de Recursos Hídricos do Pontal do Paranapanema, no intuito de minimizar diversos impactos ambientais decorrentes de ações, sobretudo, antrópicas. Além disso, recomenda-se o uso dos dados de cursos d'água e nascentes para a delimitação das APP's conforme recomendações da legislação vigente sobre o tema.

\section{REFERÊNCIAS BIBLIOGRÁFICAS}

BRAGA et al. Introdução à Engenharia Ambiental. São Paulo, Pearson Prentice Hall, 2005.

CÂMARA, G.; DAVIS, C. Introdução. In: CÂMARA, G. et al. Introdução à Ciência da Geoinformação. INPE, 2004. Cap. 1.

COMITÊ DE BACIAS HIDROGRÁFICAS DE PRESIDENTE PRUDENTE (CBH-PP). Características gerais da UGRHI-22. 2015 Disponível em: <http://paranapanema.org/ugrh/comites/sp/cbhpp/caracterizacao/>. Acesso em: 27 junho 2015. 


\section{ANAP

DERISIO, J. C. Introdução ao controle de poluição ambiental. São Paulo. Signus Editora, 2007.

EMPLASA - Empresa Paulista de Planejamento Metropolitano SA. Produtos cartográficos: projeto mapeia São Paulo - Ortofotos digitais. Disponível em: http://www.emplasa.sp.gov.br/emplasa/EmplasaEleicao/ortofotos.asp>. Acesso em: $17 \mathrm{Jul}$. 2015.

LEAL, A. C. Planejamento ambiental de bacias hidrográficas como instrumento para 0 gerenciamento de recursos hídricos. Entre-lugar, v. 3, n. 6, p. 65-84, 2012.

MEDEIROS, J. S.; CÂMARA, G. Geoprocessamento para projetos ambientais. In: CÂMARA, G. et al. Introdução à Ciência da Geoinformação. INPE, 2004. Cap. 10.

MOTA, S. Urbanização e meio ambiente. Rio de Janeiro: ABES. 2003.

ROSS, J.L.S. Ecogeografia do Brasil - Subsídios para planejamento ambiental. São Paulo, Oficina de Textos, 2009.

SANTOS, R. F. Planejamento ambiental: teoria e prática. São Paulo: Oficina de Textos. 2004. 